\section{The definition of remission in psoriatic arthritis: can this be accurate without assessment of multiple domains?}

We read with great interest the recent publication by Schoels et $a l^{1}$ outlining response criteria and disease state cut-offs for the disease activity in psoriatic arthritis (DAPSA) score. The DAPSA is simple and quick to perform in clinic, making it feasible for use in clinical practice, but we remain concerned about these new definitions: first, we have major concerns about the limitations of just assessing the joints in such a heterogeneous condition; second, the development of these cut-offs was done entirely based on physician opinion, without any inclusion of patients' views; finally, there is a concern about response criteria developed almost exclusively within randomised controlled trial (RCT) datasets, which are not representative of the psoriatic arthritis (PsA) population as a whole.

As stated in the paper, the DAPSA includes a number of key outcomes designated by the Group for Research and Assessment of Psoriasis and Psoriatic Arthritis/Outcomes in Rheumatology Clinical Trials group, but does not cover them all. Currently, the key outcome measures recommended are peripheral joint disease, psoriasis, pain, global disease activity, function and quality of life. $^{2}$ The DAPSA covers just three of these.

This is particularly concerning when developing definitions of remission for potential use in a treat-to-target strategy. Even in the expert survey where patients were classified into disease states, doctors were only given information included in the DAPSA. There was no information on entheseal, axial or skin involvement, which could radically affect expert's classification. In the discussion, the authors state that the exclusion of other musculoskeletal manifestations is a limitation, but that this is 'reasonable' 'given the relatively low frequency of entheseal involvement'. ${ }^{1}$ Most RCTs in PsA report enthesitis present in $25 \%-79 \% \%^{3}$ of their patients, depending on entheseal indices used, and similarly high rates $(79 \%)$ are seen in early PsA as seen in the Tight Control of Psoriatic Arthritis (TICOPA) study. ${ }^{5}$

Our principal concern is the development of definitions of disease state, particularly the definition of remission. Remission is a definite term implying absence of disease, ${ }^{6}$ which is not accurate using these cut-offs as mild active joint disease could be present within the score of 4 , and other aspects of the disease could be missed entirely with such a limited assessment. If this definition is used in a treat-to-target strategy, it could lead to dangerous undertreatment of patients due to misclassification. The authors suggest that other manifestations should be assessed separately, but in that case, and given the statistical advantage of composite measures, surely these outcome measures should be combined to create a definition of remission.

There is always a difficult balance between comprehensive assessment of a variable disease and feasibility of outcome measures particularly in clinical practice. However, if the authors suggest that additional measures should be used for other disease manifestations then essentially they are advocating a composite disease activity measure, such as the Composite Psoriatic Disease Activity Index (CPDAI) or Psoriatic arthritis Disease Activity Score (PASDAS), which combines individual measures. $^{7}{ }^{8}$ The suggested combination with other outcome measures also reduces the feasibility of such a measure as the DAPSA as it still requires assessment (appropriately given the heterogeneity of the disease) of multiple domains, which will increase assessment time in clinic.
Schoels et al argue against the use of existing composite measures assessing multiple domains particularly as some therapies may work well for one domain of disease, but not for others. While this has not been a common experience with any of the recently tested therapies in PsA (tumour necrosis factor (TNF) inhibitors, interleukin (IL)-17 inhibitors, IL-12/23 inhibitors), it is clear that the individual components of a composite score, such as the CPDAI or PASDAS, could be reported identifying differential responses across domains of disease.

The methodology for development of these cut-offs was robust with appropriate sensitivity analyses to test their accuracy. However, all of this was based on physician expert opinion with no input from patients. The 2010 European League Against Rheumatism recommendations, as well as many other national and international guidelines, highlight the importance of the patient's opinion with 'treatment based on a shared decision between the patient and the rheumatologist'. ${ }^{9}$ Other definitions of disease activity states and responses in PsA have been developed or subsequently validated against patient opinion. ${ }^{8}{ }^{10}$ It is disappointing that patients were not involved in the development of these cut-offs, but is key in the future that their opinion is sought as to whether these are valid.

The other key question moving forward is whether these cut-offs have prognostic impact. Does achieving 'remission' despite potentially having some element of active peripheral joint disease result in an abrogation of joint damage progression and functional decline?

Finally, there is the issue of the response rates developed in the paper. There is no clear reasoning behind the 'matching' of response rates to the ACR20/50/70 cut-offs rather than either patient or physician opinion of minor, moderate or major responses. They are developed and validated almost entirely using RCT datasets from TNF inhibitor studies. It is well recognised that the populations in these studies are not representative of the PsA population as a whole with predominant polyarticular disease typically affecting around 20 joints. ${ }^{11}$ There are no data to suggest that these response criteria would remain valid in other subtypes of disease. We recognise the inherent difficulties in assessment of PsA given its complex and variable presentation. However, in the TICOPA trial, a composite measure of minimal disease activity was used to guide treatment strategies with significant benefit to clinical and patient-reported outcomes. $^{5}$ This measure includes assessment of many more domains of disease, but remains feasible for completion in 5-10 min during the consultation. Moving forward, we have to balance accurate assessment with practical and feasible assessments to ensure that patients are treated optimally.

\section{Philip S Helliwell, Laura C Coates}

Leeds Institute of Rheumatic and Musculoskeletal Medicine, University of Leeds, Leeds, UK

Correspondence to Dr Philip S Helliwell, Leeds Institute of Rheumatic and Musculoskeletal Medicine, University of Leeds, Leeds LS7 4SA, UK; p.helliwell@ leeds.ac.uk

Competing interests None declared.

Provenance and peer review Not commissioned; internally peer reviewed.

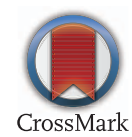

To cite Helliwell PS, Coates LC. Ann Rheum Dis 2015;74:e66. 
Received 1 September 2015

Accepted 2 September 2015

Published Online First 25 September 2015

\section{G Linked}

http://dx.doi.org/10.1136/annrheumdis-2015-208521

Ann Rheum Dis 2015;74:e66. doi:10.1136/annrheumdis-2015-208509

\section{REFERENCES}

1 Schoels MM, Aletaha D, Alasti F, et al. Disease activity in psoriatic arthritis (PsA): defining remission and treatment success using the DAPSA score. Ann Rheum Dis Published Online First: 12 Aug 2015 doi:10.1136/annrheumdis-2015-20750

2 Gladman DD, Mease PJ, Strand V, et al. Consensus on a core set of domains for psoriatic arthritis. J Rheumatol 2007:34:1167-70.

3 Antoni CE, Kavanaugh A, Kirkham B, et al. Sustained benefits of infliximab therapy for dermatologic and articular manifestations of psoriatic arthritis: results from the infliximab multinational psoriatic arthritis controlled trial (IMPACT). [erratum appears in Arthritis Rheum 2005;52:2951]. Arthritis Rheum 2005;52:1227-36.
4 Kavanaugh A, Mclnnes I, Mease P, et al. Golimumab, a new human tumor necrosis factor alpha antibody, administered every four weeks as a subcutaneous injection in psoriatic arthritis: Twenty-four-week efficacy and safety results of a randomized, placebo-controlled study. Arthritis Rheum 2009;60:976-86.

5 Coates LC, Moverley AR, McParland L, et al. Results of a randomised controlled tria comparing tight control of early psoriatic arthritis (TICOPA) with standard care: tight control improves outcome. Lancet in press.

6 Kavanaugh A, Fransen J. Defining remission in psoriatic arthritis. Clin Exp Rheumatol 2006;24(6 Suppl 43):S-83-7.

7 Mumtaz A, Gallagher P, Kirby B, et al. Development of a preliminary composite disease activity index in psoriatic arthritis. Ann Rheum Dis 2011;70:272-7.

8 Helliwell PS, FitzGerald 0, Fransen J, et al. The development of candidate composite disease activity and responder indices for psoriatic arthritis (GRACE project). Ann Rheum Dis 2013;72:986-91.

9 Gossec L, Smolen JS, Gaujoux-Viala C, et al. European League Against Rheumatism recommendations for the management of psoriatic arthritis with pharmacological therapies. Ann Rheum Dis 2012;71:4-12.

10 Coates LC, FitzGerald O, Gladman DD, et al. MDA criteria for PsA show good correlation with physician and patient opinion and proposed composite measures. Ann Rheum Dis 2012;71(Suppl 3):575.

11 Taylor WJ, Fellow DE, Helliwell PS. Case definition of psoriatic arthritis. Lancet 2000;356:2095; author reply 6 . 